\title{
Predictores de una conducta sustentable en estudiantes de pregrado de la Universidad de La Serena, Chile
}

\author{
Segundo R. Cabana, Ricardo A. Zamarreño, Rodrigo P. Véliz y Mauricio J. Sierra \\ Universidad de La Serena, Facultad de Ingeniería, Dpto. de Ing. Industrial, Casilla 554, La Serena-Chile \\ (correo-e: rcabana@userena.cl; rzamarre@userena.cl; rveliz@evascopes.com; msierra@muffinsoftware.com)
}

Recibido Mar. 25, 2020; Aceptado May. 26, 2020; Versión final Jul. 15, 2020, Publicado Dic. 2020

\begin{abstract}
Resumen
Esta investigación tiene como propósito desarrollar un modelo multivariante que permita explicar y analizar la conducta sustentable de los estudiantes de pregrado de la Universidad de La Serena (ULS), Chile. Se propone un modelo relacional con siete hipótesis de trabajo, el cual se estudió utilizando el modelo de ecuaciones estructurales (SEM, por sus siglas en inglés) por el método de mínimos cuadrados parciales (PLS, por sus siglas en inglés). Se ratifica, con confiabilidad estadística, que una conducta sustentable de los estudiantes es consecuencia de la influencia simultánea de cinco variables: desafíos del entorno, valores organizacionales, normas institucionales, conocimiento ambiental y conducta ambiental. Estas variables influyen positiva, directa e indirectamente, explicando en un $57 \%$ la varianza de la conducta. Se concluye que los resultados confirman las hipótesis y el modelo planteado.
\end{abstract}

Palabras clave: desafíos del entorno; conciencia ambiental; conducta sustentable, ecuaciones estructurales

\section{Sustainable behavior predictors in undergraduate students at the University of La Serena, Chile}

\begin{abstract}
The purpose of this research study is to develop a multivariate model that allows explaining and analyzing sustainable behavior of undergraduate students at the University of La Serena (ULS), Chile. A relational model with seven working hypotheses is proposed, which was assessed using the structural equation model (SEM) by the partial least squares (PLS) method. The results are statistical reliable and confirm that student sustainable behavior is a consequence of the simultaneous influence of five variables: environmental challenges, organizational values, institutional norms, environmental knowledge, and environmental behavior. These variables showed a positive direct and indirect influence, accounting for $57 \%$ of the behavior's variance. In conclusion, the results confirm the hypotheses and the proposed model.
\end{abstract}

Keywords: environmental challenges; awareness; sustainable behavior; structural equations 


\section{INTRODUCCIÓN}

El desequilibrio climático producido por la crisis medioambiental que enfrentamos actualmente, se ha convertido en el mayor desafío que la humanidad ha enfrentado, colocando todos los esfuerzos, tanto de los gobiernos como de la comunidad científica, en reducir los gases de efecto invernadero que emanan de la actividad humana. El desbalance climático que el planeta está enfrentando, tiene como resultado un aumento significativo en el nivel del mar, sequías muy prolongadas, incendios devastadores producidos por las altas temperaturas, como los vividos por Australia el 2019, cambios en la tendencia de las precipitaciones, disminución de las capas de nieve y hielo, entre otros efectos que no solo afectan las actividades productivas del ser humano, sino que colocan entre dicho su propia existencia.

A nivel nacional, las regiones de todo el país ya están siendo afectadas por los impactos del cambio climático, de una manera que difiere según su ubicación geográfica, impactos que se reflejan en sus actividades productivas, su vulnerabilidad económica, social y ambiental y otra serie de factores que en conjunto determinan finalmente el riesgo al cual se ven enfrentadas. Esto hace necesario el liderazgo de los diferentes actores relevantes que permitan, de manera conjunta, intervenir positivamente en la profunda relación existente entre las dimensiones de la crisis ambiental y los modos y estrategias del comportamiento humano y la organización social Corraliza (2001).

Es por ello, que es un desafío estratégico formar personas que asuman un rol activo en la generación y ejecución de soluciones que reduzcan sistemáticamente las problemáticas sociales y ambientales, ejecutando e incentivando acciones destinadas a conservar la integridad de los recursos sociofísicos de nuestro planeta, es decir personas con conductas sustentables (Corral et al., 2011). Para esto, las instituciones de educación superior deben liderar en base al ejemplo, con planes de estudio que aborden temas de la sociedad y de perspectiva ambiental, educando y entregando el conocimiento ambiental necesario para el desarrollo de un entorno adecuado que promueva la participación de los estudiantes (Cuzdriorean et al., 2020; Gifford y Nilson, 2014). Este entorno, con información, valores y normas establecidas, tiene el potencial de cambiar la identidad personal, la visión del mundo y los valores de los estudiantes para que estos posean una conciencia y conductas más sustentables (Dagiliūtè et al., 2018).

Por ende, un factor crucial para cuidar, revertir o mitigar los daños causados, así como también para conservar y preservar el medio ambiente, es la educación, específicamente la educación ambiental. Este proceso de enseñanza-aprendizaje debe de desarrollar la conciencia, vinculando la conexión emocional con el medio, para el cuidado de la naturaleza (Vliegenthart et al., 2016). Al desarrollar esta conciencia ambiental en las personas, se potenciará su conexión con el medio ambiente a través de un equilibrio consciente simbiótico, considerando el conocimiento, los afectos, las experiencias, las actitudes y la conducta (Zylstra et al., 2014).

Es por ello que es necesario estimular a los estudiantes a que también asuman un rol de "cliente-estudiante", para quien la satisfacción y participación protagónica en los procesos de enseñanza-aprendizaje es imprescindible (Cabana et al., 2016). Si bien, una línea de investigación se opone a ver al estudiante como "cliente", dada su noción de Gestión de la Calidad Total (TQM), que busca garantizar la satisfacción del estudiante como un "cliente", lo que implicaría complacer sus demandas a corto plazo y llevarlos a culpar a la institución de sus fracasos. Sin embargo, esta percepción está fuera de sintonía con las teorías contemporáneas de cliente, donde estos son responsables de su propia satisfacción, incluso, en esta analogía los estudiantes solo se encuentran satisfechos cuando obtienen una educación de calidad en el campo de su elección y con un diploma que se valora en el mercado laboral (Mark, 2013; Kotze y du Plessis, 2003; Royo, 2017). En consecuencia, se debe buscar que los estudiantes asuman un rol activo protagónico en los centros de educación superior, donde deben formarse técnica y valóricamente, para que desarrollen una conducta sustentable que les permita generar soluciones proactivas de problemáticas sociales y ambientales. Así será factible pasar desde las normas (políticas) institucionales a adquirir conocimientos ambientales que lo lleven a asumir una conciencia ambiental y una conducta sustentable. En esta transformación, el rol de las Universidades es clave, dado sus modelos educativos, donde sus estudiantes deben ser protagonistas del cambio, por ello el objetivo de esta investigación es identificar los predictores de la conducta sustentable de estudiantes de pregrado de la Universidad de La Serena - Chile.

\section{OTROS ANTECEDENTES}

Bajo el contexto anterior, la presente investigación propone un modelo causal basado en siete hipótesis relacionadas con los conceptos de: 1) desafíos del entorno; 2) valores organizacionales; 3) normas institucionales; 4) conocimiento ambiental; 5) conducta ambiental; y 6) conducta sustentable. Las relaciones de estas variables, y en consecuencia el modelo causal propuesto presente en la Figura 1, se basa en la siguiente fundamentación teórica. 


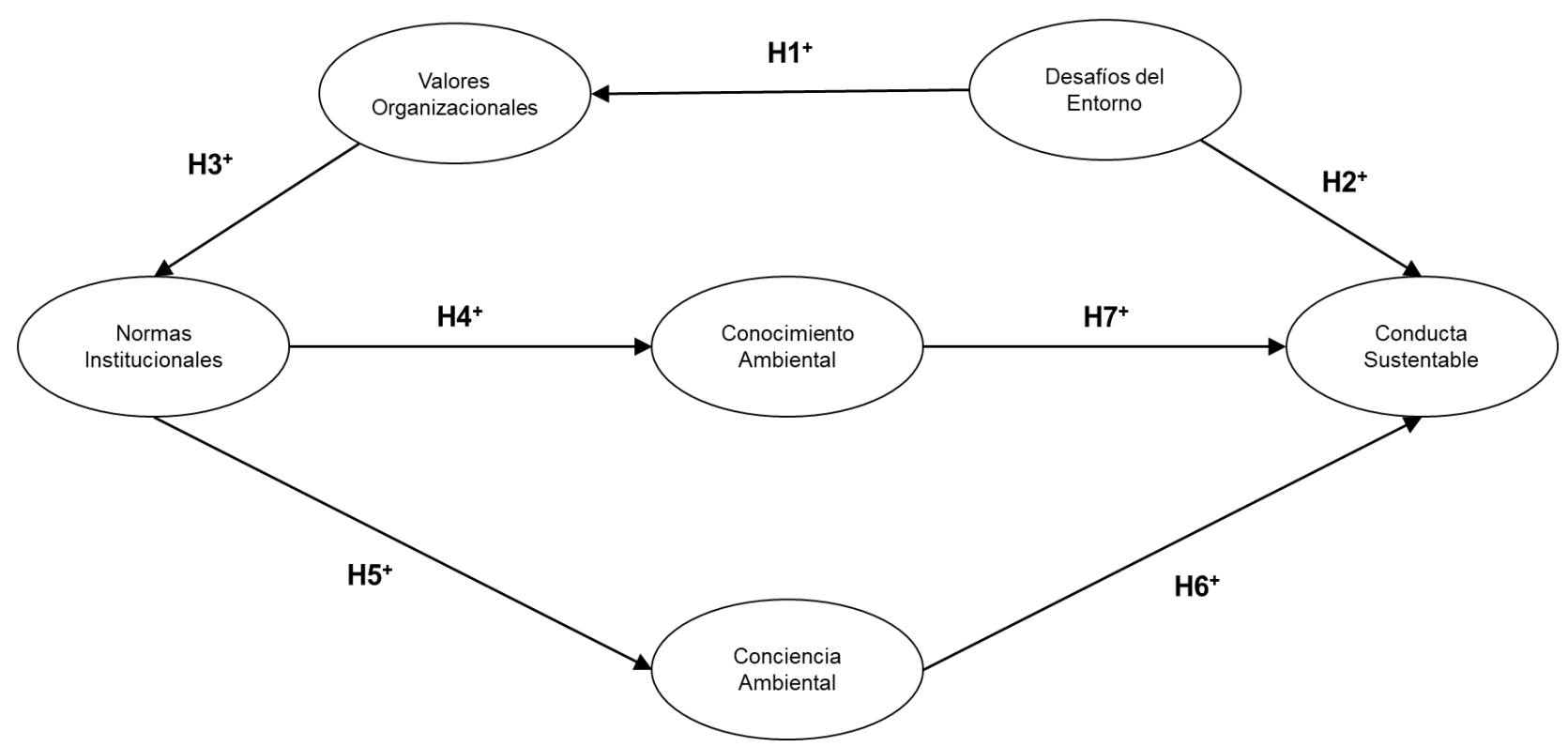

Fig. 1: Modelo causal hipotetizado

\section{Desafíos del entorno, valores organizacionales y conducta sustentable}

La sociedad contemporánea se enfrenta a nuevos retos relacionados con la gestión del ambiente, dado que actualmente se sufren las consecuencias del proceso de industrialización acaecido en las últimas décadas (Espino et al., 2015). Y como es sabido, las organizaciones son sistemas abiertos muy susceptibles a los cambios del entorno, por lo cual su transformación interna, depende en gran medida, de los cambios sociales, culturales y económicos que se generan en el exterior. En esta línea, el compromiso con la sustentabilidad, como un valor organizativo, tiende a formarse, en mayor medida, como consecuencia del entorno. Para las organizaciones, estudiar los factores de su entorno es una necesidad, ya que de esta forma se logra estar preparado y tener una estrategia para combatir los cambios que se presentan en las industrias y la sociedad, con ello, se logra generar un mayor compromiso, de forma tal que las personas con sus decisiones y/o conducta logran los objetivos planteados, para dar respuesta a los desafíos del entorno (Ávila, 2017).

Dada esta problemática ambiental, al igual que las organizaciones, el entorno en el cual se ven insertos los estudiantes, modifica la conducta del mismo a través de normas sociales que influyen significativamente en su comportamiento, proporcionándole información importante sobre su entorno y comprensión de qué conductas prevalecen y son recompensadas, así como también qué conductas son raras y castigadas (Poškus, 2016). En este contexto, la familia como integrante de la sociedad, también es parte de los desafíos y normas del entorno, mostrando a sus miembros lo que se espera de ellos, condicionada principalmente por las directrices y requerimientos culturales provenientes del sistema social en el que se desenvuelve (valores culturales, creencias, sucesos históricos, familia extensa, trabajo, amistades) (Cortes et al., 2017).

El rol del estado, como parte del entorno, es de suma importancia, pues no sólo deberá diseñar un marco regulatorio para el desarrollo del medio ambiente y la sociedad, sino que, además, deberán crear políticas públicas de sustentabilidad, para incentivar a las organizaciones y por ende a las personas a ser constantes creadoras de valor compartido, un atributo relevante en la gestión sustentable (Cabana y Pino, 2019).

\section{Valores organizacionales y normas institucionales}

Los valores plantean el marco ético - social dentro del cual la empresa lleva a cabo sus acciones, formando parte de la cultura organizacional y estableciendo los límites en los cuales deben enmarcarse la conducta de los individuos que pertenecen a ella, tanto en lo organizacional como en lo personal (Vidal y Pérez, 2016). Los factores de la cultura organizacional tales como la misión, visión, valores y políticas, sí influyen significativamente en el compromiso organizacional con la sustentabilidad (Carro et al., 2017). Además, se puede plantear que las actitudes de las personas, son consecuencia de los valores y normas organizacionales que la preceden, y es una tendencia evaluadora (ya sea positiva o negativa) con respecto de personas, hechos o cosas, según Pérez y Borrazá (2012). Complementariamente, se hace necesario potenciar el liderazgo estratégico en organizaciones educacionales que tengan como propósito instalar conductas ambientales en sus estudiantes, pues así se posibilitará el logro de una visión donde los valores proambientales serán centrales y además se instalarán las capacidades para gestionar con eficacia el entorno (Cortes et al., 2017), y por ende se instalarán normas que lo posibilitarán. 


\section{Normas institucionales, conocimiento y conciencia ambiental}

Los valores de una unidad educativa se convierten en principios de acción, normas que influirán en los conocimientos y conciencia ambiental de los estudiantes. Por supuesto, es necesario buscar caminos con una mirada de trabajo cooperativo entre los miembros de la comunidad educacional; directivos, profesores, padres y estudiantes, que lleven a mejorar la conducta ambiental de los estudiantes. Como complemento, se requiere mejorar el espacio en el cual se desarrollan las actividades pedagógicas, colocar acento en la importancia y los beneficios que reporta el conservar todo limpio y ordenado, como también respetar y cuidar los recursos naturales con que se cuenta, incrementando así la base valórica de los estudiantes (Cortes et al.,2017), por ende, potenciando su conciencia ambiental.

Los gestores de organizaciones, deberían incluir en su planificación acciones que potencien el conocimiento de los individuos sobre el medio ambiente, normas que orienten los valores que guían la vida de las personas y sus intenciones, para que estas se comporten de un modo más ecológico y responsable (López et al., 2015). Incorporar la sustentabilidad en las organizaciones, les otorga características que promueven la transparencia y la rendición de cuentas con sus stakeholders (partes interesadas), con el cumplimiento de los acuerdos establecidos y la resolución de conflictos, atributos que dotan de una mayor competitividad a la organización (Arredondo et al., 2014).

Marquez et al. (2011) identifican que las actitudes de los estudiantes son positivas para aprender prácticas sustentables, así como la importancia que tiene la escuela como fuente de información ambiental, lo que ciertamente es determinante tanto en la motivación para aprender y por ende en su conciencia ambiental. Complementariamente, Zamorano et al., (2011), plantean que los estudiantes de educación superior tienen la capacidad de identificar la problemática ambiental actual, así también comprenden los daños que ocasiona la contaminación en general.

\section{Conocimiento ambiental y conducta sustentable}

En los últimos 40 años las instituciones de educación superior han promovido e incentivado el involucramiento de sus estudiantes en conductas sustentables dentro de los campus mediante programas, integrados a su plan de estudio, destinados a proporcionar el conocimiento suficiente para convertirse en ciudadanos sustentables una vez se gradúen y abandonen la institución (Cogut et al., 2019). En este contexto, la educación es crucial para la entrega del conocimiento que permita el desarrollo de la conciencia, valores y actitudes necesarias para lograr un desarrollo sustentable (Biasutti y Frate, 2017), conocimiento que influye significativamente en la conducta sustentable de los estudiantes, haciendo de estas instituciones, los actores principales en el desarrollo del intelecto humano y su conducta (Scott, 2013; Mojilis, 2019).

Olsson et al., (2019), plantean que el comportamiento sustentable de los estudiantes se ve afectado si se experimenta una enseñanza pluralista en la que se fomente el comportamiento pro social. De igual forma, en una investigación realizada en Chile, con adultos jóvenes, Neaman et al., (2018) encontraron que un comportamiento prosocial y un comportamiento proambiental poseen similares facetas y son parte del mismo sistema, llegando a la conclusión de que trabajar con un comportamiento prosocial en educación también afectará el comportamiento proambiental. Cheng y Monroe (2012), añaden la importancia de experiencias pasadas en la naturaleza, la proximidad del lugar de residencia a la naturaleza, el conocimiento ambiental como predictores de la motivación de participar de actividades ambientalmente responsables.

\section{Conciencia ambiental y conducta sustentable}

Álvarez y Vega (2009), señalan que la educación ambiental tiene como objetivo guiar a la sociedad al desarrollo sustentable, por lo que debe facilitar que las personas puedan, comprender y ser conscientes de la complejidad de los problemas ambientales a nivel global. Para promover una vida más sostenible, la educación ambiental tiene que enseñar actitudes, conocimientos, valores y comportamientos desde la conciencia. Esta conciencia "ambiental" se desarrolla según Corraliza (2001), cuando acciones basadas en un conjunto integrado de valores, creencias y regulaciones permiten disminuir un impacto negativo de la acción humana en el medio ambiente. Otros autores señalan que, para desarrollar conciencia ambiental, las emociones deben estar vinculadas al reconocimiento de problemas ambientales que permiten el inicio o mantenimiento de estos problemas vinculados al comportamiento de las personas (Álvarez y Vega, 2009).

En el mismo ámbito, estudios comparativos demuestran que el desarrollo de conciencia ambiental juega un papel fundamental en la protección del medio ambiente, las políticas ciudadanas y paralelamente en la economía, se considera que los ingresos y la contaminación, juegan un rol fundamental en el desarrollo de la educación ambiental (Xudong et al., 2019), y por ende son la base para desarrollar en las personas una conducta sustentable. 
Por ello, la actitud ambiental, entendida como una conciencia ambiental está compuesto por un conjunto de factores psicológicos específicos relacionados con la propensión de los individuos para llevar a cabo comportamientos proambientales o ecológicos (Aguilar et al., 2012). Complementaria-mente, se reconoce la importancia que los estudiantes le otorgan al medio ambiente para disfrutar de un mundo mejor, por ello la contaminación y destrucción de los recursos naturales son percibidos como los principales problemas del entorno en el que viven (Espino et al., 2015), condición central para asumir una conducta sustentable coherente con la conciencia ambiental que han expresado. Dado el sustento teórico anterior, es posible declarar las siguientes siete hipótesis de investigación detalladas en la Tabla 1.

Tabla 1: Hipótesis de investigación

\begin{tabular}{|l|l|l|}
\hline Nombre & Código & Descripción \\
\hline Hipótesis 1 & H1 & Desafíos del Entorno influye directa y positivamente en los Valores Organizacionales. \\
\hline Hipótesis 2 & H2 & Desafíos del Entorno influye directa y positivamente en la Conducta Sustentable. \\
\hline Hipótesis 3 & H3 & Valores Organizacionales influye directa y positivamente en las Normas Institucionales. \\
\hline Hipótesis 4 & H4 & Normas Institucionales influye directa y positivamente en el Conocimiento Ambiental. \\
\hline Hipótesis 5 & H5 & Normas Institucionales influye directa y positivamente en la Conciencia Ambiental. \\
\hline Hipótesis 6 & H6 & Conciencia Ambiental influye directa y positivamente en la Conducta Sustentable. \\
\hline Hipótesis 7 & H7 & Conocimiento Ambiental influye directa y positivamente en la Conducta Sustentable. \\
\hline
\end{tabular}

\section{MÉTODOLOGÍA}

Esta sección presenta el procedimiento metodológico utilizado para la validación del modelo anteriormente propuesto. Además, se indica la fiabilidad de los indicadores, validez de cada constructo y su validez discriminante permitiendo dilucidar la pertinencia de los indicadores y constructos con respecto al modelo.

\section{Muestra}

Se aplica una encuesta a todas las Facultades de la Universidad de La Serena, El tamaño conocido de la población es 7.404 estudiantes. La muestra se distribuye según las Facultades de Ciencias (21,7\%), Ciencias Sociales y Económicas (19,2\%), Ingeniería (37,5\%) y Humanidades $(21,7 \%)$ con un tamaño de 366 y un nivel de confianza del $95 \%$ (5\% de error). La Tabla 2 presenta un resumen de la distribución de estudiantes encuestados por nivel cursado y Facultad.

Tabla 2: Descripción facultad según nivel

\begin{tabular}{|l|l|l|l|l|l|l|l|}
\hline Facultad & 1 año & 2 año & 3 año & 4 año & 5 año & 6 año & Total \\
\hline Facultad de Ciencias & 35 & 14 & 21 & 6 & 3 & 0 & 79 \\
\hline Facultad de Ciencias Sociales y Económicas & 23 & 37 & 6 & 4 & 0 & 0 & 70 \\
\hline Facultad de Ingeniería & 42 & 32 & 28 & 7 & 12 & 17 & 138 \\
\hline Facultad de Humanidades & 34 & 22 & 4 & 9 & 10 & 0 & 79 \\
\hline Tamaño de la muestra & 134 & 105 & 59 & 26 & 25 & 17 & 366 \\
\hline
\end{tabular}

\section{Procedimiento}

La recolección de datos se realizó a través de una encuesta presencial a estudiantes de las diferentes facultades de la Universidad de La Serena (ULS) entre marzo y mayo del 2019, realizando un muestreo aleatorio estratificado con afijación proporcional. La investigación de carácter exploratorio utiliza la técnica estadística SEM (Structural Equation Modeling), la cual fue aplicada mediante el software estadístico Smart PLS. Esta técnica permite determinar si los constructos e indicadores cumplen con los análisis de fiabilidad y validez, además de determinar las cargas factoriales también llamados indicadores PATH, los que, finalmente demuestran si las hipótesis son aceptadas o de lo contrario son rechazadas.

Cuestionario y fiabilidad individual de los indicadores

El cuestionario consiste en 31 preguntas que se agrupan en 5 constructos: desafíos del entorno, valores organizacionales, normas institucionales, conocimiento ambiental, conciencia ambiental y conducta sustentable. Las preguntas son politómicas tipo Likert con puntuación o categorías de 1 a 5 . Para determinar la fiabilidad individual se obtuvo la carga factorial individual de cada uno de los indicadores señalados en la encuesta, esto es posible ya que el índice Kaiser-Meyer-Olkin (KMO) fue superior a 0,5 para cada uno de los 
constructos y la prueba de esfericidad de Bartlett $(P E B)$ es significativa $(p<0,05)$ según Méndez y Rodón (2012) (ver Tabla 2). Para este cálculo se utilizó el software SPSS Statistics 23 utilizando la opción de reducción de dimensiones, el cual entregó los resultados observados. Los autores plantean distintos valores mínimos para aceptar un indicador como componente integrador de un constructo. Una regla adecuada en este caso, se considera que el ítem es un indicador del factor cuando su carga factorial sea mayor a 0.4, siempre que la carga en el resto de los factores sea menor que este valor. En la Tabla 3, se aprecian todos los constructos, indicadores y carga factorial asociada.

Tabla 3: Carga factorial por indicador según su constructo.

\begin{tabular}{|c|c|c|c|}
\hline Constructo & Indicador & Ítem & $\begin{array}{l}\text { Carga } \\
\text { Factorial }\end{array}$ \\
\hline \multirow{10}{*}{$\begin{array}{l}\text { Desafíos del Entorno } \\
\text { KMO: } 0,727 \\
\text { PEB: } 0,000\end{array}$} & DE1 & Problemáticas sociales y ambientales nacionales & 0,811 \\
\hline & DE2 & Problemáticas sociales y ambientales regionales & 0,574 \\
\hline & DE3 & Calidad de vida de la región & 0,612 \\
\hline & DE4 & Tendencia de mercado social y ambiental & 0,757 \\
\hline & DE5 & Comunidades empoderadas pro ambiental y social & 0,66 \\
\hline & DE6 & Entorno familiar pro Ambiental y social & 0,612 \\
\hline & DE7 & Conciencia de colaboración & 0,64 \\
\hline & DE8 & Trato igualitario & 0,762 \\
\hline & DE9 & Influencia de la gobernanza ambiental & 0,564 \\
\hline & DE10 & Desafíos de inclusión & 0,65 \\
\hline \multirow{4}{*}{$\begin{array}{l}\text { Valores Organizacionales } \\
\text { KMO: } 0,518 \\
\text { PEB: } 0,00\end{array}$} & VO1 & Principios pro ambientales y sociales & 0,725 \\
\hline & VO2 & Universidad de La Serena (ULS) responsable & 0,766 \\
\hline & VO3 & Modelo educativo con sello social y ambiental & 0,716 \\
\hline & VO4 & Valores consistentes con foco social y ambiental & 0,744 \\
\hline \multirow{5}{*}{$\begin{array}{l}\text { Normas Institucionales } \\
\text { KMO: } 0,544 \\
\text { PEB: } 0,000\end{array}$} & NI1 & Políticas incentivan uso ecoeficiente & 0,757 \\
\hline & $\mathrm{NI} 2$ & Normas que visibilizan el sello social y ambiental & 0,589 \\
\hline & $\mathrm{NI3}$ & $\begin{array}{l}\text { Normas que impulsan programas y acciones académicas } \\
\text { ambientales y sociales }\end{array}$ & 0,796 \\
\hline & $\mathrm{NI} 4$ & Políticas que premian conductas sustentables & 0,654 \\
\hline & $\mathrm{NI5}$ & Normas que aseguran financiamiento plurianual & 0,585 \\
\hline \multirow{3}{*}{$\begin{array}{l}\text { Conocimiento Ambiental } \\
\text { KMO: } 0,623 \\
\text { PEB: } 0,000\end{array}$} & CA1 & $\begin{array}{l}\text { Programas académicos conectados con los desafíos } \\
\text { ambientales y sociales }\end{array}$ & 0,793 \\
\hline & CA2 & Redes sociales visibilizan la prioridad social y ambiental & 0,912 \\
\hline & CA3 & Espacios formales de educación Ambiental & 0,256 \\
\hline \multirow{5}{*}{$\begin{array}{l}\text { Conciencia Ambiental } \\
\text { KMO: } 0,598 \\
\text { PEB: } 0,000\end{array}$} & $\mathrm{Cm} 1$ & Valores me motivan a ser responsable con el entorno & 0,842 \\
\hline & $\mathrm{Cm} 2$ & Tengo un perfil ecológico & 0,641 \\
\hline & $\mathrm{Cm3}$ & Mis actitudes me llevan a colaborar y pensar en el bien común & 0,798 \\
\hline & $\mathrm{Cm} 4$ & $\begin{array}{l}\text { Mis principios me llevan a respetar las expectativas de la } \\
\text { comunidad }\end{array}$ & 0,79 \\
\hline & Cm5 & $\begin{array}{l}\text { Mis creencias me motivan a integrarme ecológicamente en mi } \\
\text { espacio cercano }\end{array}$ & 0,768 \\
\hline \multirow{5}{*}{$\begin{array}{l}\text { Conducta Sustentable } \\
\text { KMO: } 0,580 \\
\text { PEB: } 0,000\end{array}$} & CS1 & $\begin{array}{l}\text { Mis acciones periódicas son responsables con el medio } \\
\text { ambiente y la sociedad }\end{array}$ & 0,482 \\
\hline & CS2 & Cuido los recursos energéticos & 0,78 \\
\hline & CS3 & Practico acciones de reciclaje & 0,736 \\
\hline & CS4 & $\begin{array}{l}\text { Ejecuto iniciativas que llevan a ser una Universidad } \\
\text { sustentable }\end{array}$ & 0,794 \\
\hline & CS5 & Me relaciono periódicamente con respeto y solidaridad & 0,883 \\
\hline
\end{tabular}

\section{Fiabilidad y validez del constructo}

Se evalúa la validez de las escalas utilizadas (validez convergente), para lo cual se analiza la varianza extraída media (AVE). Se recomienda que esta sea superior a 0,5 con lo que se establece que más del $50 \%$ de la varianza del constructo se debe a sus indicadores (Hameed y Abdul, 2014). Todos los constructos entregan un AVE superior al mínimo esperado, por lo que se comprueba la validez convergente de éstos (ver Tabla 3). Finalmente, la validez discriminante indica en qué medida un constructo es diferente a los otros que conforman el modelo. Una forma de comprobar este criterio de validación, es demostrar que las correlaciones entre los constructos son más bajas que la raíz cuadrada de la varianza extraída media AVE (Hameed y Abdul, 2014). Como se observa en la Tabla 4, todos los valores cumplen para la validez discriminante. 
Tabla 4: Índices de fiabilidad y validez del constructo.

\begin{tabular}{|l|l|l|l|}
\hline & Alfa de Cronbach & $\begin{array}{l}\text { Fiabilidad } \\
\text { compuesta }\end{array}$ & $\begin{array}{l}\text { Varianza } \\
\text { extraída media } \\
\text { (AVE) }\end{array}$ \\
\hline Desafíos del Entorno & 0,734 & 0,849 & 0,652 \\
\hline Valores Organizacionales & 0,653 & 0,842 & 0,729 \\
\hline Normas Institucionales & 0,789 & 0,875 & 0,700 \\
\hline Conocimiento Ambiental & 0,755 & 0,837 & 0,563 \\
\hline Conciencia Ambiental & 0,758 & 0,846 & 0,579 \\
\hline Conducta Sustentable & 0,672 & 0,813 & 0,594 \\
\hline
\end{tabular}

\section{Validez discriminante}

La validez discriminante indica en qué medida un constructo es diferente a los otros que conforman el modelo. Una forma de comprobar este criterio de validación, es demostrar que las correlaciones entre los constructos son más bajas que la raíz cuadrada de la varianza extraída media AVE. Como se observa en la Tabla 5, todos los valores cumplen para la validez discriminante.

Tabla 5: Índice de Validez Discriminante del constructo.

\begin{tabular}{|l|l|l|l|l|l|l|}
\hline Constructo & $D E$ & VO & CA & NI & Cm & CS \\
\hline Desafíos del Entorno & 0,808 & & & & & \\
\hline Valores Organizacionales & 0,002 & 0,854 & & & & \\
\hline Normas Institucionales & 0,024 & 0,527 & 0,837 & & & \\
\hline Conocimiento Ambiental & 0,141 & 0,370 & 0,505 & 0,750 & & \\
\hline Conciencia Ambiental & 0,145 & 0,342 & 0,569 & 0,461 & 0,761 & \\
\hline Conducta Sustentable & $-0,021$ & 0,310 & 0,370 & 0,353 & 0,541 & 0,771 \\
\hline
\end{tabular}

\section{RESULTADOS}

Luego de realizar los análisis de confiabilidad y validez del modelo propuesto, se utilizará el método de modelos estructurales a través del Método de Mínimos Cuadrados Parciales (Partial Least Squares, PLS), debido a que este método está orientado a modelos de carácter predictivo y exploratorio, en donde el conocimiento teórico no está del todo desarrollado, como es el caso el modelo propuesto en la Figura 1. El utilizar este método, facilita confirmar las relaciones existentes entre las variables y la aceptación o rechazo de las hipótesis propuestas.

\section{Validación del modelo estructural}

Una vez estudiada la validez del modelo de medida, a continuación, se evaluarán las relaciones causales que se proponen en el modelo, por medio de dos índices básicos: la varianza explicada (R2) y los coeficientes path o pesos de regresión estandarizados $(\beta)$. La varianza explicada de las variables endógenas o dependientes (R2) debe ser igual o mayor que 0,1 (Falk y Millar, 1992). A partir de este criterio empírico todos los constructos poseen una calidad de poder de predicción aceptable, como se puede observar en la Tabla 3 los constructos cumplen con este criterio. Otra prueba que determina la calidad de predicción de modelo estructural es el Test de Stone-Geisser (Q2). Este test se usa como criterio para medir la relevancia predictiva de los constructos dependientes y se calcula por medio de la técnica Blindfolding. En el caso de que la Q2>0, indica que el modelo tiene relevancia predictiva. En caso contrario, no la tiene (Chin, 1998). Como se puede observar en la Tabla 6, en todos los casos los valores de Q2 son positivos, lo cual certifica la relevancia predictiva del modelo.

Tabla 6: Validación del modelo estructural por medio de su varianza explicada $\left(R^{2}\right)$ y el test de Stone-Geisser $\left(Q^{2}\right)$

\begin{tabular}{|l|l|l|}
\hline Constructo & $R^{2}$ & $Q^{2}$ \\
\hline Valores Organizacionales & 0,560 & 0,012 \\
\hline Normas Institucionales & 0,511 & 0,135 \\
\hline Conocimiento Ambiental & 0,571 & 0,156 \\
\hline Conciencia Ambiental & 0,482 & 0,051 \\
\hline Conducta Sustentable & 0,574 & 0,271 \\
\hline
\end{tabular}




\section{Índice de bondad de ajuste (GoF)}

Según autores como Esposito, Trinchera, Squillacciotti y Tenenhaus (2008) sugieren un criterio global de bondad de ajuste para los modelos estructurales PLS. Estos autores proponen que el índice de bondad de ajuste global se dé por medio de la raíz cuadrada de la multiplicación de la media aritmética del análisis de la varianza extraída (AVE) y la media aritmética de la varianza explicada (R2) de las variables endógenas o dependientes. Como se puede observar en la Tabla 7, el índice de bondad de ajuste (GoF) del modelo de análisis es de 0,57 , lo cual demuestra que se tiene un buen ajuste en el modelo de medida y en el modelo estructural, cumpliendo así con el criterio empírico de que la medida de bondad de ajuste debe variar entre 0 y 1, a mayor valor, mejor será el índice (Tenenhaus, 2008).

Tabla 7: Índice de bondad de ajuste (GoF)

\begin{tabular}{|l|l|l|l|}
\hline & AVE & $\mathrm{R}^{2}$ & GoF \\
\hline Desafíos del Entorno & 0,652 & & \\
\hline Valores Organizacionales & 0,729 & 0,560 & \\
\hline Normas Institucionales & 0,700 & 0,511 & \\
\hline Conocimiento Ambiental & 0,563 & 0,571 & \\
\hline Conciencia Ambiental & 0,579 & 0,482 & \\
\hline Conducta Sustentable & 0,594 & 0,574 & \\
\hline Media Aritmética & 0,636 & 0,525 & 0,57 \\
\hline
\end{tabular}

\section{Contraste de Hipótesis}

Los coeficientes path, coeficientes de regresión parcial que representan el efecto de una variable sobre otra, o pesos de regresión estandarizados ( $\beta$ ), los valores deseables deberían estar por encima de 0,3 siendo 0,2 un valor mínimo aceptable (Chin, 1998). Como se observa en la Tabla 8 los paths todas las relaciones propuestas cumplen con la regla de estar sobre el mínimo valor aceptado. Por otro lado, el error estándar de los parámetros debe verificar la condición del estadístico T>=1,96 (Kwong, 2013).

Tabla 8: Contraste de las hipótesis. En la Tabla, ${ }^{* *}$ significa t-value $>1,96$ ( $p$-value $\left.<0,05\right)$

\begin{tabular}{|l|l|l|l|l|}
\hline Hipótesis & Relaciones & Paths $(\beta)$ & $\begin{array}{l}\text { Valor } T \\
(\text { Bootstrap })\end{array}$ & Contraste \\
\hline H1 & D. del Entorno $\rightarrow$ V. Organizacionales & 0,352 & $4.327^{* *}$ & Acepta \\
\hline H2 & D. del Entorno $\rightarrow$ Conducta Sustentable & 0,714 & $17,042^{* *}$ & Acepta \\
\hline H3 & V. Organizacionales $\rightarrow$ N. Institucionales & 0,483 & $5.476^{* *}$ & Acepta \\
\hline H4 & N. Institucionales $\rightarrow$ Conocimiento Amb. & 0,551 & $7,674^{* *}$ & Acepta \\
\hline H5 & N. Institucionales $\rightarrow$ Conciencia Amb. & 0,374 & $6,265^{* *}$ & Acepta \\
\hline H6 & Conciencia Amb. $\rightarrow$ Conducta Sustentable & 0,632 & $9,428^{* *}$ & Acepta \\
\hline
\end{tabular}

\section{DISCUSIÓN}

Se ratifica con confiabilidad estadística que una conducta sustentable de los estudiantes de pregrado de la Universidad de La Serena, posee predictores que actúan simultáneamente, cinco variables que influyen positiva, indirecta e indirectamente en ella; desafíos del entorno, conciencia ambiental, conocimiento, normas institucionales y valores organizacionales, que explican en un $57,4 \%$ la varianza de la conducta sustentable, que es un nivel significativo según Medina et al., (2012). En consecuencia, esas variables son el pilar fundamental para incentivar un cambio tanto a nivel institucional, como también, de la misma comunidad universitaria, potenciando la participación efectiva en programas e iniciativas ambientales.

Respecto a la influencia individual de los constructos sobre la conducta sustentable, el constructo desafíos del entorno es la que tiene un mayor impacto positivo, seguido por conciencia y conocimiento ambiental, pues presentan un coeficiente estandarizado de $0.71,0,63$ y 0.48 , respectivamente. Esto evidencia con confiabilidad estadística que los desafíos ambientales y sociales que se instalan en la región, a nivel nacional o internacional, influyen determinantemente en las conductas sustentables que asumen los estudiantes, de allí que las normas o políticas institucionales que formalmente representan una guía que la ULS espera que sus estudiantes consideren para actuar y decidir, es menos relevante. Específicamente, estas normas explican mejor la varianza del conocimiento ambiental que poseen los estudiantes $(57,1 \%)$, que a su conciencia ambiental (48,2\%), varianzas que tienen un nivel significativo según Medina et al. (2012). 
Las normas institucionales, considerando los coeficientes estandarizados, influyen más en conocimiento ambiental que poseen los estudiantes $(0,55)$, que en la conciencia ambiental que ellos(as) realmente internalizan $(0,37)$. De allí que los estudiantes respetan el "marco regulatorio" que la Universidad busca instalar, aprenden de ese conocimiento ambiental y actúan consistentemente. Esta influencia es menor en comparación con la significancia de los desafíos del entorno y la conciencia ambiental que preferentemente se forman a nivel familiar. Así el reto es ejecutar efectivamente las normas institucionales, una ejecución que debe ser pertinente al contexto de una universidad del Estado, pues con ello se logrará que la comunidad universitaria (las personas, normas institucionales y su arquitectura organizacional), sean determinantes en el desarrollo de una conducta sustentable. También, se desprende que las normas institucionales deben impulsar la implementación de programas o iniciativas corporativas y académicas que se ejecutan para "construir" una Universidad sustentable. Asegurándose que su liderazgo y dirección impacte positivamente en la conciencia ambiental, entendiendo que es menos complejo internalizar conocimiento ambiental.

Para cumplir con el desafío anterior, es necesario posicionar de forma más efectiva el rol ambiental y social que los estudiantes deben asumir en el proceso de pregrado, ejerciendo y desarrollando así un liderazgo sustentable. Activo que tiene el potencial para provocar los cambios de valor que se requieren a nivel de la sociedad, la universidad, los estudiantes y los funcionarios académicos y no académicos de un centro de educación superior. El liderazgo sustentable, entendido como un gestor del cambio, que expresen los estudiantes, será determinante para su empleabilidad en el mercado laboral, dado los requerimientos de la sociedad y una economía globalizada, cada vez más conectada con la economía del bien común. Complementariamente, será significativo para el crecimiento integral de los estudiantes, como una persona empoderada que debe contribuir con el desarrollo sostenible y sustentable de su universidad y la sociedad en general.

Así la institución debe fortalecer y dar más pertinencias a sus normativas y programas relacionados con educación ambiental y sustentabilidad, comunicándolas y asignándole un presupuesto plurianual adecuado a nivel corporativo, y asegurándose que se ejecuten continua y sistemáticamente a través de sus funcionarios, académicos y no académico. Integrando en el proceso a los estudiantes con un rol activo, que les permita ir paulatinamente internalizando conocimiento y conciencia ambiental que serán la base de una conducta sustentable.

Se expresa que la varianza de los valores organizacionales (corporativos) de la ULS, es explicada importantemente por los desafíos del entorno (56,0\%). Así los directivos y la comunidad universitaria formulan valores que están adecuadamente en sintonía con su entorno local y global, reflejando con ello una Universidad que se "construye" consistentemente con las demandas de los stakeholders que son relevantes para su desarrollo sostenible y sustentable. Sin embargo, dada la baja influencia de las normas o políticas institucionales en la conciencia ambiental de los estudiantes y desde allí en su conducta sustentable, se identifica como desafío estratégico para la ULS implementar los ajustes en el sistema de liderazgo y la misma arquitectura organizacional que le den valor a dichas normas institucionales, desde las percepciones y posición de los estudiantes, académicos y funcionarios no académicos.

Pero no solo basta con esfuerzos individuales o valores y normas institucionales bien diseñadas y difundidas en los medios corporativos, es necesario el compromiso irrestricto de la institución en su conjunto, para evolucionar de las buenas intenciones a programas y acciones efectivamente ejecutados, logrando así resultados de valor a nivel triestamental. En esta evolución, se llegará a un estado deseado, donde una conducta sustentable estará presente cotidianamente en los estudiantes de pregrado, será un hábito, que impulsará la creación de un ecosistema que movilizará a la comunidad triestamental, a liderar los cambios que se deben instalar a nivel de la arquitectura organizacional de la ULS.

\section{CONCLUSIONES}

De los resultados, sus análisis y discusión se pueden extraer las siguientes conclusiones sobre la conducta sustentable de los estudiantes de la ULS:

1) Es posible confirmar las hipótesis planteadas, es decir, los desafíos del entorno, valores organizacionales, normas institucionales, conocimiento ambiental y conciencia ambiental son significativas y están positivamente relacionadas con la conducta ambiental;

2) Se ratifica la importancia estratégica de las instituciones de educación superior en la misión de formar estudiantes con un perfil orientado a la sustentabilidad;

3) Se determina que los desafíos del entorno juegan un rol fundamental en la conducta sustentable, dando así, un incentivo en el desarrollo más específico de este constructo. En esta línea será interesante ahondar más profundamente en la participación de la familia, amigos y entorno digital en el que están insertos los estudiantes y como estos influyen en su conducta sustentable. 


\section{AGRADECIMIENTOS}

Los autores agradecen el apoyo entregado por la Universidad de La Serena para terminar exitosamente esta investigación.

\section{REFERENCIAS}

Aguilar, M.C., García, J.M., Calvo, A., y Salinas, J.M., Comparative Study Between the Theory of Planned Behavior and the Value-Belief-Norm Model Regarding the Environment, on Spanish Housewives' Recycling Behavior, https://doi.org/10.1111/j.1559-1816.2012.00962.x, G Journal Of Applied Social Psychology, 42(11), 2797-2833 (2012)

Álvarez, P. y Vega, P., Actitudes Ambientales y Conductas Sostenibles para la Educación Ambiental. Revista Psicodidáctica, 14(2), 245-260 (2009).

Arredondo, F.G., De la Garza, J.G. y Parra, J.C., Transparencia en las Organizaciones, una Aproximación desde la Perspectiva de los Colaboradores, https://doi.org/10.1016/j.estger.2014.06.007, Estudios Gerenciales,30(133), 408-418 (2014).

Ávila, V.S., Desafíos del Sector Primario y Políticas Públicas Sustentables, https://doi.org/10.1016/j.ecin.2017.01.003, Economía Informa, 402, 29-39 (2017).

Biasutti, M., y Frate, S., A validity and Reliability Study of the Attitudes Toward Sustainable Development Scale, https://doi.org/10.1080/13504622.2016.1146660, Environmental Education Research, 23(2), 214-230 (2016)

Cabana, S.R., Cortés, F.H., Vega, D.L. y Cortés, R.A., Análisis de la Fidelización del Estudiante de Ingeniería con su Centro de Educación Superior: Desafíos de Gestión Educacional, http://dx.doi.org/10.4067/S0718-50062016000600009, Formación Universitaria, 9(6), 93-104 (2016).

Cabana, S., Pino, C., Modelo de Gerencia Sustentable para Fortalecer la Competitividad en las Empresas del Retail "Supermercados" de la Región de Coquimbo, Chile, http://dx.doi.org/10.22201/fca.24488410e.2020.2007, Contaduría y Administración, próxima publicación (2019).

Carro, J., Sarmiento, S. y Rosano, G., La Cultura Organizacional y su Influencia en la Sustentabilidad Empresarial. La Importancia de la Cultura en la Sustentabilidad Empresarial, http://dx.doi.org/10.1016/j.estger.2017.11.006, Estudios Gerenciales,33(145), 352-365 (2017).

Cheng, J.C. y Monroe, M.C., Connection to Nature: Children's Affective Attitude Toward Nature, https://doi.org/10.1177/0013916510385082, Environment and Behavior, 44, 31-49 (2012).

Chin, W., The Partial Least Squares Approach to Structural Equation Modelling; in Modern Methods for Business Research, Lawrence Erlbaum Associates Publishers, 295 - 336, Mahwah, New Jersey, USA (1998).

Cogut, G., Webster, N. J., Marans, R. W., y Callewaert, J., Links Between Sustainability-Related Awareness and Behavior, https://doi.org/10.1108/IJSHE-09-2018-0161, International Journal of Sustainability in Higher Education, 20(7), 1240-1257 (2019)

Corral V., Montiel, M.M. y otros cuatro autores, Psychological Wellbeing as Correlate of Sustainable Behaviors, International Journal of Hispanic Psychology, ISSN: 1939-5841, 4(1), 31-44 (2011)

Corraliza, J.A., El Comportamiento Humano y los Problemas Ambientales, https://doi.org/10.1174/021093901609541, Estudios de Psicología, 22(1), 3-9 (2001).

Cortes, F., Cabana, R. y otros tres autores, Variables influyentes en la Conducta Ambiental en Alumnos de Unidades Educativas, Región de Coquimbo-Chile, http://dx.doi.org/10.4067/S0718-07052017000200002, Estudios Pedagógicos, 43(2), 27-46 (2017).

Cuzdriorean, D.D., Fekete, S., y Vladu, A.B., Identifying the Promoters of Students Sustainable Behaviour: An Empirical Study, Amfiteatru Economic, 22(54), 432-446 (2020).

Dagiliūtè, R., Liobikienè, G., y Minelgaitè, A., Sustainability at Universities: Students' Perceptions from Green and NonGreen Universities, https://doi.org/10.1016/j.jclepro.2018.01.213, Journal of Cleaner Production, 181, 473-482 (2018)

Espino, P., Olaguez, E. y Davizon, Y., Análisis de la Percepción del Medio Ambiente de los Estudiantes de Ingeniería en Mecatrónica, http://dx.doi.org/10.4067/S0718-50062015000400006, Formación Universitaria, 8(4), 45-54 (2015).

Esposito, V., Trinchera, L., Squillacciotti, S. y Tenenhaus, M., REBUS-PLS: A Response-Based Procedure for Detecting Unit Segments in PLS Path Modelling, Applied Stochastic Models in Business and Industry, 24(5), 439-458 (2008).

Falk, R. y Miller, N., A Primer for Soft Modeling, 1를., University of Akron Press, Akron, USA (1992).

Gifford, R. and Nilsson, A., Personal and Social Factors that Influence Pro- Environmental Concern and Behaviour: $A$ Review, https://doi.org/10.1002/ijop.12034, International Journal of Psychology, 49(3), 141-157 (2014)

Hameed, A., e I. Abdul, SEM-PLS Analysis of Inhibiting Factors of Cost Performance for Large Construction Projects in Malaysia: Perspective of Clients and Consultants, https://doi.org/10.1155/2014/165158, The Scientific World Journal (2014) 
Kotzé, T.G., y du Plessis, P.J., Students as "Co-Producers" of Education: a Proposed Model of Student Socialisation and Participation at Tertiary Institutions, https://doi.org/10.1108/09684880310501377, Quality Assurance in Education, 11(4), 186-201 (2003)

Kwong, K., Partial Least Squares Structural Equation Modeling (PLS-SEM) Techniques Using SmartPLS. Marketing Bulletin, 24, 1-32 (2013).

López, M., Álvarez, P. y González, E., Conocimiento, Valores e Intenciones como Determinantes del Comportamiento Ecológico, http://dx.doi.org/10.3989/ris.2015.73.3.e018, Revista Internacional De Sociología, 73(3), 1-11 (2015).

Mark, E., Student Satisfaction and the Customer Focus in Higher Education, https://doi.org/10.1080/1360080X.2012.727703, Journal of Higher Education Policy and Management, 35(1), 2-10 (2013).

Marquez, R., Salavarría, O. y otros seis autores, Cultura ambiental en estudiantes de Bachillerato. Estudio de Caso de la Educación Ambiental en el Nivel Medio Superior de Campeche, Revista Electrónica De Investigación Educativa, ISSN 1607-4041, 13(2), 84-98 (2011).

Medina, B., López de Llergo, L. y Díaz, A., La Medición de Datos Cualitativos, una Tendencia en Investigación Social: Análisis del Caso de la Facultad de Contaduría y Administración, Unidad Culiacán, Ra Ximhai, ISSN: 1665-0441, 8(2),287-295 (2012).

Méndez, C. y Rondón, M., Introducción al Análisis Factorial Exploratorio, Revista colombiana de Psiquiatría, ISSN: $0034-$ 7450, 41(1), 197-207 (2012).

Mojilis, F., Sustainability Awareness of Students from a Green University in Sabah, Malaysia, Journal of Tourism, Hospitality and Environment Management, eISSN: 0128-178X, 4(13), 24-33 (2019)

Neaman, A., Otto, S., y Vinokur, E., Toward an Integrated Approach to Environmental and Prosocial Education, https://doi.org/10.3390/su10030583, Sustainability, 10(3), 583 (2018).

Olsson, D., Gericke, N., Boeve-de Pauw, J., Berglund, T. y Chang, T., Green schools in Taiwan - Effects on Student Sustainability Consciousness, https://doi.org/10.1016/j.gloenvcha.2018.11.011, Global Environmental Change, 54, 184194 (2019).

Pérez, D. y Borrazás, R., Aproximación a la Concepción de Valores Compartidos en la Dirección Estratégica de una Institución Universitaria, Revista Varona, (5), 12-17 (2012).

Royo, J., Students as Customers: a Paradigm Shift in Higher Education, https://doi.org/10.28939/iam.debats-en.2017-11, DEBATS. Journal on Culture, Power and Society, 131(3), 137-149 (2017)

Scott, W., Developing the Sustainable School: Thinking the Issues Through, https://doi.org/10.1080/09585176.2013.781375, The Curriculum Journal, 24(2), 181-205 (2013)

Tenenhaus, M., Component-Based Structural Equation Modelling, https://doi.org/10.1080/14783360802159543, Total Quality Management Journal \& Business Excellence, 79(7/8), 871-886 (2008)

Vidal, M. y Pérez, A., Formación en Valores. Conceptos Éticos y Tecnológicos, Métodos y Estrategias, Revista Cubana de Educación Médica Superior, ISSN 1561-2902 ,10(18), 81-94 (2016)

Vliegenthart, A., Corcuera, E., y Quezada, M., Educación Ambiental al Aire Libre (EAAL) como Metodología para la Conservación de la Biodiversidad, Educación (2016).

Xudong, C., Bihong, H. y Chin-Te, L., Environmental Awareness and Environmental Kuznets Curve, https://doi.org/10.1016/j.econmod.2019.02.003, Economic Modelling, 77, 2-11 (2019).

Zamorano, B., Parra V. y otros tres autores, Compromiso Ambiental de los Estudiantes del Nivel Medio Superior, Revista Desarrollo Local Sostenible, Red Académica Iberoamericana Local Global, ISSN: 1988-5245, 4(11), 1-13 (2011).

Zylstra, M.J., Knight, A.T., Esler, K.J. y Le Grange, L.L., Connectedness as a Core Conservation Concern: An Interdisciplinary Review of Theory and a Call for Practice, https://doi.org/10.1007/s40362-014-0021-3, Springer Science Reviews, 2(1), 119-143 (2014). 
